Journal of Computer Science 3 (11): 841-846, 2007

ISSN 1549-3636

(C) 2007 Science Publications

\title{
An Improved Implementation of Brain Tumor Detection Using Segmentation Based on Neuro Fuzzy Technique
}

\author{
${ }^{1} \mathrm{~S}$. Murugavalli and ${ }^{2} \mathrm{~V}$. Rajamani \\ ${ }^{1}$ Department of Computer Science and Engineering \\ ${ }^{2}$ Department of Electronics and Communication Engineering, \\ PSNA College of Engineering and Technology, \\ Dindigul, Tamil Nadu, India
}

\begin{abstract}
Implementation of a neuro-fuzzy segmentation process of the MRI data is presented in this study to detect various tissues like white matter, gray matter, csf and tumor. The advantage of hierarchical self organizing map and fuzzy c means algorithms are used to classify the image layer by layer. The lowest level weight vector is achieved by the abstraction level. We have also achieved a higher value of tumor pixels by this neuro-fuzzy approach. The computation speed of the proposed method is also studied. The multilayer segmentation results of the neuro fuzzy are shown to have interesting consequences from the viewpoint of clinical diagnosis. Neuro fuzzy technique shows that MRI brain tumor segmentation using HSOM-FCM also perform more accurate one.
\end{abstract}

Key words: Image analysis, segmentation, HSOM, FCM, neuro fuzzy technique, tumor detection

\section{INTRODUCTION}

Magnetic Resonance Imaging (MRI) is the state-ofthe-art medical imaging technology which allows cross sectional view of the body with unprecedented tissue contrast $^{[1-2]}$. MRI plays an important role in assessing pathological conditions of the ankle, foot and brain. It has rapidly evolved into an accepted modality for medical imaging of disease processes in the musculoskeletal system, especially the foot and brain due to the use of non-ionizing radiation. MRI provides a digital representation of tissue characteristic that can be obtained in any tissue plane. The images produced by an MRI scanner are best described as slices through the brain. MRI has the added advantage of being able to produce images which slice through the brain in both horizontal and vertical planes.

Segmentation is an important process to extract information from complex medical images. Segmentation has wide application in medical field ${ }^{[3-4]}$. The main objective of the image segmentation is to partition an image into mutually exclusive and exhausted regions such that each region of interest is spatially contiguous and the pixels within the region are homogeneous with respect to a predefined criterion. Widely used homogeneity criteria include values of intensity, texture, color, range, surface normal and surface curvatures. During the past many researchers in the field of medical imaging and soft computing have made significant survey in the field of image segmentation ${ }^{[5-8]}$. Image segmentation techniques can be classified as based on edge detection, region or surface growing, threshold level, classifier such as Hierarchical Self Organizing Map (HSOM), and feature vector clustering or vector quantization. Vector quantization has proved to be a very effective model for image segmentation process ${ }^{[9]}$. Vector quantization is a process of portioning an n-dimensional vector space into $M$ regions so as to optimize a criterion function when all the points in each region are approximated by the representation vector $\mathrm{X}_{\mathrm{i}}$ associated with that region. There are two processes involved in the vector quantization: one is the training process which determines the set of codebook vector according to the probability of the input data, the other is the encoding process which assigns input vectors to the code book vectors. Vector quantization process has been implemented in terms of the competitive learning neural network $(\mathrm{CLNN})^{[10]}$. Self Organizing Map $(\mathrm{SOM})^{[11]}$ is a member of the CLNNs and this can be the best choice when implementing vector quantization using neural network ${ }^{[11-16]}$. The importance of SOM for vector quantization is primarily due to the similarity between the competitive learning process employed in the SOM and the vector quantization procedure. The main shortcoming of the SOM is that the number of

Corresponding Author: S. Murugavalli, Department of Computer Science and Engineering, PSNA College of Engineering and Technology, Dindigul, Tamilnadu, India 
neural units in the competitive layer needs to be approximately equal to the number of regions desired in the segmented image. It is not however, possible to determine a priori the correct number of regions $\mathrm{M}$ in the segmented image. This is the main limitation of the conventional SOM for image segmentation. The HSOM directly address the aforesaid shortcomings of the SOM. HSOM is the combination of self organization and topographic mapping technique. HSOM combine the idea of regarding the image segmentation process as one of data abstraction where the segmented image is the final domain independent abstraction of the input image. The hierarchical segmentation process for a hierarchical structure is called abstraction tree. The abstraction tree bears some resemblance to the major familiar quad tree data structure ${ }^{[17]}$ used in the several image processing and image analysis algorithms. Clustering is the process of grouping a data set in a way that the similarity between data within a cluster is maximized while the similarity between data of different clusters is maximized ${ }^{[18]}$ and is used for pattern recognition in image processing. To recognize a given pattern in an image various techniques have been utilized, but in general two broad categories of classifications have been made: unsupervised techniques and supervised techniques. In the unsupervised method, data items that are to be clustered are not preclassified while in supervised clustering the data points are preclassified. One of the well-known unsupervised algorithms that can be applied to many applications such as image segmentation ${ }^{[19]}$, fuzzy $\mathrm{c}$ means $(\mathrm{FCM})^{[20]}$ etc.. FCM algorithm is one of the popular fuzzy clustering algorithms which are classified as constrained soft clustering algorithm. A soft clustering algorithm finds a soft partition of a given data set by which an element in the data set may partially belong to multiple clusters. Moreover, there is a constraint on the function that the membership degree of a point in all the clusters adds up to $1^{[21-22]}$. The researchers in this field have used SOM or HSOM or FCM separately as one of the tool for the image segmentation of MRI brain for the tumor analysis. In this paper, we propose a hybrid technique combining the advantages of HSOM and FCM and implemented for the MRI image segmentation process to detect various tissues like white matter, gray matter, cst and tumor.

\section{IMPLEMENTATION OF HSOM AND FCM ALGORITHM}

In this section we discussed the implementation of the HSOM and FCM algorithm in detail. First, we describe the HSOM structure, learning procedure and then FCM with abstraction tree. The pseudocode for the above neuro fuzzy technique is also presented at the end of this section.

The HSOM is organized as pyramidal structure consisting of multiple layers where each layer resembles the single layer SOM. The detailed explanations and the structure of the HSOM were presented by S.M. Bhandarkar et.al. ${ }^{[2]}$. Learning process consists of sequential corrections of the vectors representing neurons. On every step of the learning process a random vector is chosen from the initial data set and then the best-matching (the most similar to it) neuron coefficient vector is identified. The winner is selected, which is the most similar to the input vector $^{[23]}$. The distance between the vectors usually measured in the Euclidean metric and is given by

$$
\left\|\mathrm{x}-\mathrm{W}_{\mathrm{C}}\right\|=\min _{\mathrm{i}}\left\{\left[\mathrm{x}-\mathbf{W}_{\mathrm{i}}\right]\right\}
$$

Where, $\mathrm{x}$ is the neuron, $\mathrm{W}_{\mathrm{C}}$ is the winning neuron vector and $\mathrm{W}_{\mathrm{i}}$ is the weight vector. The modified weight vector coefficients can be calculated by

$$
\mathbf{W}_{\mathrm{i}}(\mathrm{t}+1)=\mathbf{W}_{\mathrm{i}}(\mathrm{t})+\mathrm{h}_{\mathrm{ci}}(\mathrm{t}) *[\mathrm{x}(\mathrm{t})-\mathbf{W}(\mathbf{t})]
$$

Where $t$ is the epoch number (discrete-time index), $\mathrm{x}(\mathrm{t})$ is the vector and is obtained by selecting a sample randomly for iteration $t$. The function $\mathrm{h}_{\mathrm{ci}}(\mathrm{t})$ is called neighborhood function and it represents a nonincreasing function of time and the distance between the winning neuron and its neighbors on the grid. The function $\mathrm{h}_{\mathrm{ci}}(\mathrm{t})$ consists of two parts: the proper distance function and the learning rate function and is given by

$$
\mathrm{h}(\mathrm{t})=\mathrm{h}\left(\left\|\mathrm{r}_{\mathrm{c}}-\mathrm{r}_{\mathrm{i}}\right\|\right) * \mathrm{a}(\mathrm{t})
$$

where, $r$ determines neuron position on the grid. The result of neighborhood function $\mathrm{h}(\mathrm{t})$ is an initial cluster center (centroids) for fuzzy c means algorithms. Cluster is a group of vectors with the distance between any two of them shorter than that between this group and the neighboring ones.

\section{FUZZY C-MEANS ALGORITHM (FCM)}

FCM algorithm based on the concept of fuzzy Cpartition, which was introduced by various researcher in this field Ruspini ${ }^{[24]}$, developed by Dunn ${ }^{[25]}$ and generalized by Bezdek ${ }^{[26]}$. The aim of FCM is to find 
cluster centers (centroids) that minimize dissimilarity functions ${ }^{[21-22]}$. In order to accommodate the fuzzy partitioning technique, the membership matrix (U) is randomly initialized as

$$
\sum_{\mathrm{i}=1}^{\mathrm{c}} \mathrm{U}_{\mathrm{ij}}=1, \forall \mathrm{j}=1, \ldots, \mathrm{n}
$$

where, $i$ is the number of cluster and $j$ is the image data points.

The dissimilarity function can be computed as

$$
\mathrm{J}\left(\mathrm{U}, \mathrm{c}_{1}, \mathrm{c}_{2}, \ldots, \mathrm{c}_{\mathrm{c}}\right)=\sum_{\mathrm{i}=1}^{\mathrm{c}} \mathrm{J}_{\mathrm{i}}=\sum_{\mathrm{i}=1}^{\mathrm{c}} \sum_{\mathrm{j}=1}^{\mathrm{n}} \mathrm{u}_{\mathrm{ij}} \mathrm{m}_{\mathrm{i}_{\mathrm{ij}}}{ }^{2}
$$

where, $\mathrm{U}_{\mathrm{ij}}$ is between 0 and $1, \mathrm{c}_{\mathrm{i}}$ is the centroid of cluster $\mathrm{i}, \mathrm{d}_{\mathrm{ij}}$ is the Euclidian distance between $i_{\text {th }}$ centroid $\left(c_{i}\right)$ and $j_{\text {th }}$ data point, $m$ is a weighting exponent and the value is greater than one. The minimum of dissimilarity function can be computed as

$$
U_{i j}=\frac{1}{\sum_{k=1}^{c}\left(\frac{d_{i j}}{d_{k j}}\right)^{2 /(m-1)}}
$$

where, $d_{i j}=\left\|x_{i}-c_{j}\right\|, d_{k j}=\left\|x_{i}-c_{k}\right\|, x_{i}$ is the $i$ th of $d-$ dimensional data, $c_{j}$ is the d-dimension center of the cluster and $\|*\|$ is any norm expressing the similarity between any measured data and center. This iteration will stop when $\operatorname{Max}_{\mathrm{ij}}\left\{\left|\mathrm{u}_{\mathrm{ij}}{ }^{(\mathrm{k}+1)}-\mathrm{u}_{\mathrm{ij}}{ }^{(\mathrm{k})}\right|\right\}<\varepsilon$, where $\varepsilon$ is a termination criterion between 0 and 1 , whereas $k$ are the iteration steps. The steps of the FCM algorithm has been listed as follows

Initialize $\mathrm{U}=\left[\mathrm{u}_{\mathrm{ij}}\right]$ matrix, $\mathrm{U}^{(0)}$

At k-step: Initialize centers vectors $\mathrm{C}^{(\mathrm{k})}=\left[\mathrm{c}_{\mathrm{j}}\right]$ taken from HSOM mapping clustering algorithm Update $\mathrm{U}^{(\mathrm{k})}, \mathrm{U}^{(\mathrm{k}+1)}$, then compute the dissimilarity function

$$
\mathrm{U}_{\mathrm{ij}}=\frac{1}{\sum_{\mathrm{k}=1}^{\mathrm{c}}\left(\frac{\mathrm{d}_{\mathrm{ij}}}{\mathrm{d}_{\mathrm{kj}}}\right)^{2 /(\mathrm{m}-1)}}
$$

If $\left\|\mathrm{U}^{(\mathrm{k}+1)}-\mathrm{U}^{(\mathrm{k})}\right\|<\varepsilon$ then STOP; otherwise return to step 3.

In the first step, the algorithm selects the initial cluster centers from SOM clustering algorithm. Then, in later steps after several iterations of the algorithm, the final result converges to actual cluster center. Therefore a good set of initial cluster is achieved and it is very important for an FCM algorithm. If a good set of initial cluster centers is chosen, the algorithm make less iterations to find the actual cluster centers. The winning neural units and their corresponding weight vectors from each layer result in a hierarchical structure termed as an abstraction tree ${ }^{[1]}$. Each node in the abstraction tree represents the region of the image at a specified level of abstraction ${ }^{[2]}$. A segmented image is generated on demand by traversing the abstraction tree in the breadth first manner starting from the root node until some criterion is met. The size of the abstraction tree (weight vector) is expanded if the sum of the variances of weight vector divided by size of the weight vector is less than element of weight vector. Otherwise the node is labeled as a closed node and none of its descendants are visited. Regions corresponding to the closed nodes constitute a segmented image and the resulting segmented image usually contains the regions from different abstraction levels.

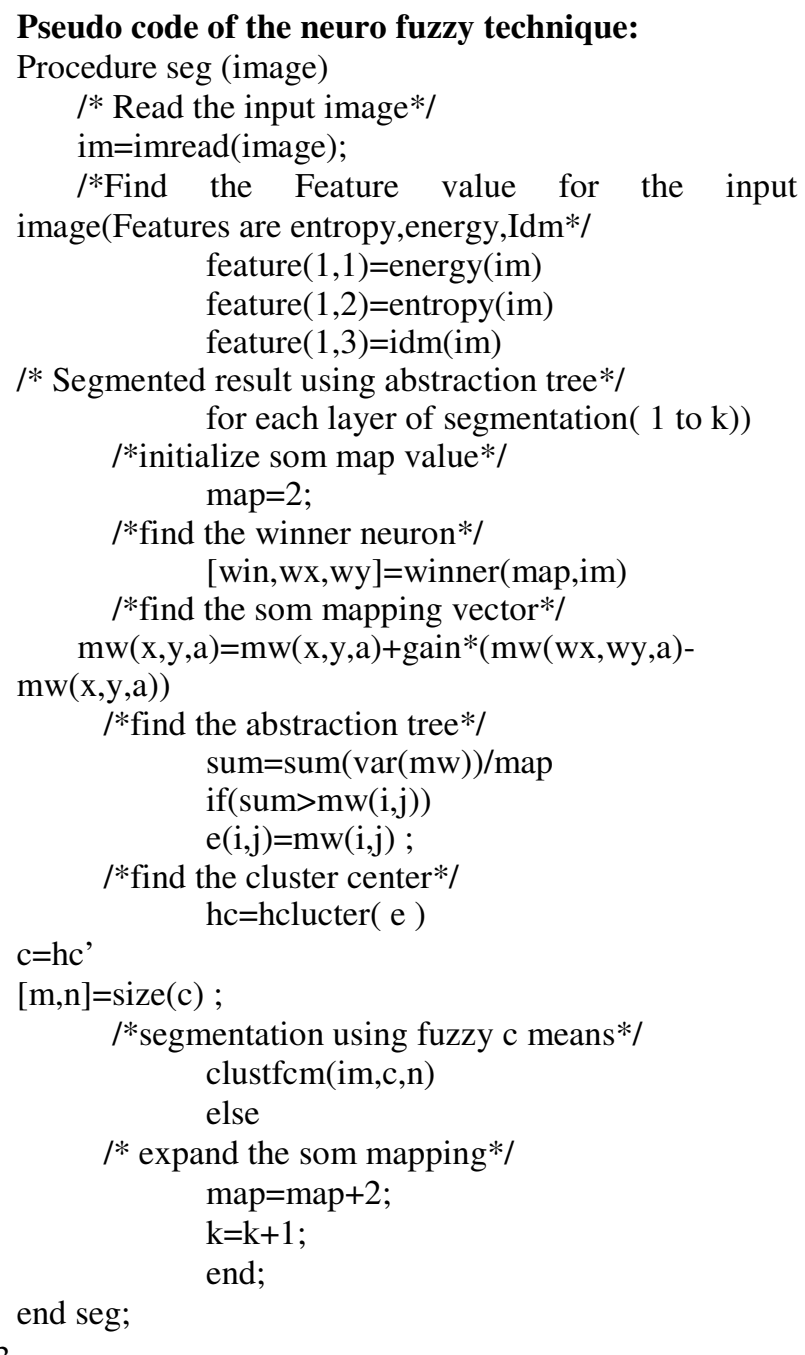

Pseudo code of the neuro fuzzy technique:

cedure seg (image)

im=imread(image);

$/ *$ Find the Feature value for the input image(Features are entropy,energy,Idm*/ feature $(1,1)=\operatorname{energy}(\mathrm{im})$ feature $(1,2)=$ entropy $(\mathrm{im})$ feature $(1,3)=\mathrm{idm}(\mathrm{im})$

/* Segmented result using abstraction tree*/ alize som map value*/ he winner neuron*/ win, wy]=winner (map,im)

/*find the som mapping vector*/ $\mathrm{x}, \mathrm{y}, \mathrm{a}))$ $\operatorname{sum}=\operatorname{sum}(\operatorname{var}(\mathrm{mw})) / \mathrm{map}$ if $(\operatorname{sum}>\operatorname{mw}(i, j))$ $\mathrm{e}(\mathrm{i}, \mathrm{j})=\mathrm{mw}(\mathrm{i}, \mathrm{j})$; hc $=$ hclucter $(\mathrm{e})$

$\mathrm{c}=\mathrm{hc}$ 


\section{RESULTS}

The results of the implementation of the hybrid neuro fuzzy segmentation process are discussed in this section. Any computer aided analysis; the execution time is one of the important parameters for analyzing medical images. The number of tumor pixels detected by various methods with the execution time is presented in Table 1. In these results, we have calculated the number of pixels affected by the tumor cells and the results have been compared with the existing results. Our proposed neuro fuzzy based segmentation technique provides better values. For the computation purpose, we have used the input features as entropy, energy and idm. The accuracy of the brain tumor segmentation process is compared with the existing methods. The percentage detection of tissues like tumor is 97.3. We have also studied the execution time for different segmentation techniques. SOM $\mathrm{k}$ means and HSOM $\mathrm{k}$ means require less time than the proposed one. The weight vector value obtained for the proposed method is less compared to the existing results. This is due to the clustering process and abstraction level technique.

The variation of the weight vector values for the different types of segmentation process is depicted in Fig .1. The weight vector for the SOM with fuzzy is $8 \times 8$. The weight vector for the SOM with $\mathrm{k}$ means is about $12 \times 12$. This weight vector value for the SOM with fuzzy is higher ${ }^{[1]}$ but it is less in our proposed method. The weight vector for the HSOM with $\mathrm{k}$ means and HSOM with fuzzy are also depicted in the above Fig. 1. The weight vector for the HSOM with $\mathrm{k}$ means is means. The input features for the segmentation process $^{[1]}$ are mean, median, standard deviation.

The input features for the calculation of the weight vector for the HSOM with c means includes some new feature like energy, entropy and idm. We have used six features, but we have achieved the lowest value of weight vector values. This is due to the fuzzy clustering technique and the abstraction level.

Figure 2 shows the variation of the total number of tumor pixels detected of an image with various segmentation techniques. The value of the tumor cells

Table 1: Weight vector value, detected tumor pixels and execution time with segmentation

\begin{tabular}{llll}
\hline $\begin{array}{l}\text { Types of } \\
\text { segmentation }\end{array}$ & $\begin{array}{l}\text { Value of } \\
\text { weight vector }\end{array}$ & $\begin{array}{l}\text { Total No. } \\
\text { Tumor value(pixel) }\end{array}$ & $\begin{array}{l}\text { Execution } \\
\text { Time }(\mathrm{sec})\end{array}$ \\
\hline SOM-kmean $^{[1]}$ & 12 & 2772 & 24.98 \\
SOM-fuzzy & 8 & 3223 & 93.39 \\
HSOM-kmean $^{[1]}$ & 12 & 2772 & 45.63 \\
HSOM-fuzzy $^{\text {HSOM }}$ & 6 & 3223 & 100.03 \\
\hline
\end{tabular}

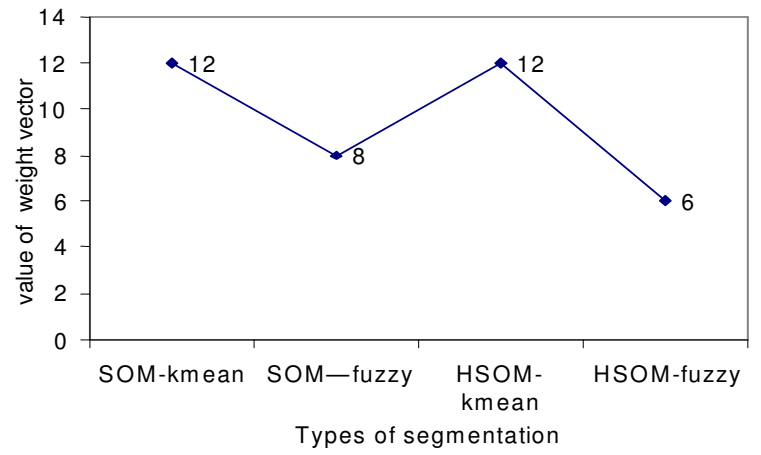

Fig. 1: Relationship between the weight vector with the types of segmentation techniques

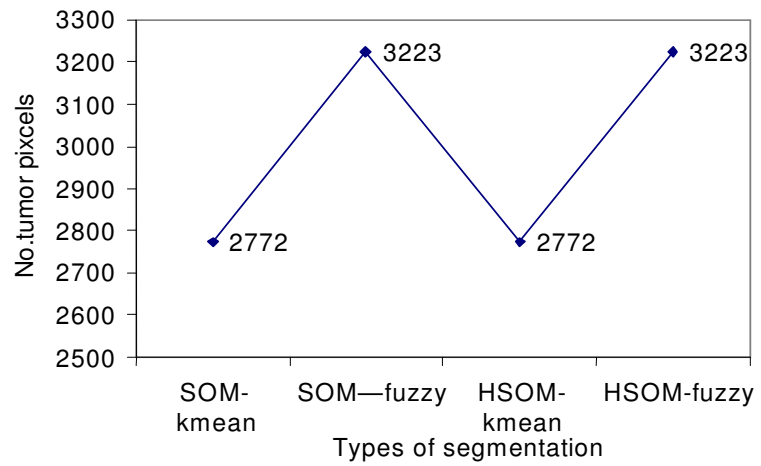

Fig. 2: Variation of total number of tumor pixels with type segmentation techniques

detected with our proposed implementation is about 3223 for the both HSOM and SOM with fuzzy but the value of the tumor pixel detected for the SOM $\mathrm{k}$ means and HSOM k means is only 2772 . We have used the standard input feature are mean, median and standard deviation for the SOM and HSOM with $\mathrm{k}$ means. In our proposed implementation of the neuro fuzzy technique the input features are entrophy, energy, idm in addition to the above standard input features. The increase in the value of the detected tumor cells is due to the abstraction level and fuzzy clustering process.

In image processing, execution time is an important parameter to analyse any image in general and in medical image is particular. Variation of the execution time for various segmentation processes is depicted in Fig.3. The execution time for the SOM with FCM is 93.39 seconds as against 24.98 seconds and HSOM with FCM is 100.03 seconds as against 100.03 seconds. The increase in the execution time for the proposed implementation is due to the layer by layer abstraction level and fuzzy clustering techniques. 


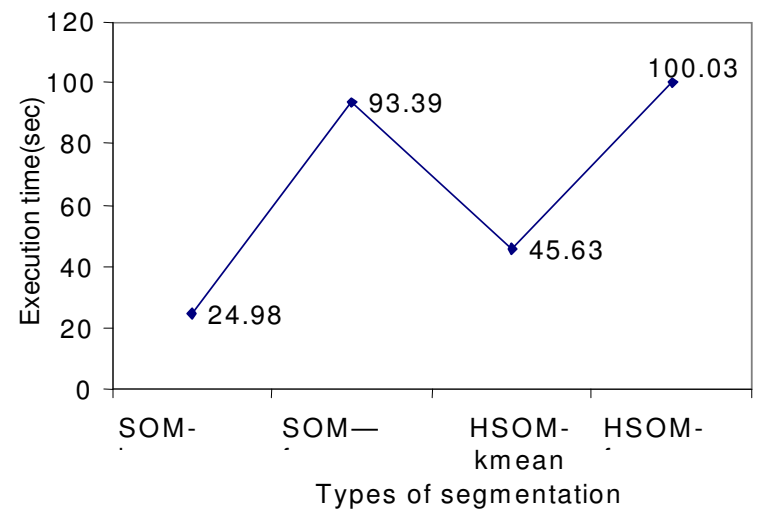

Fig. 3: Variation of execution time with types of segmentation

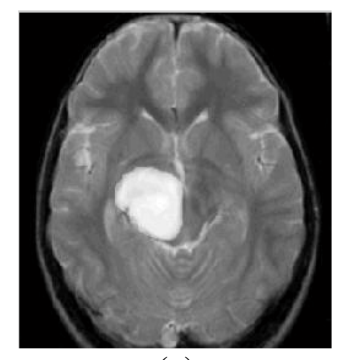

(a)

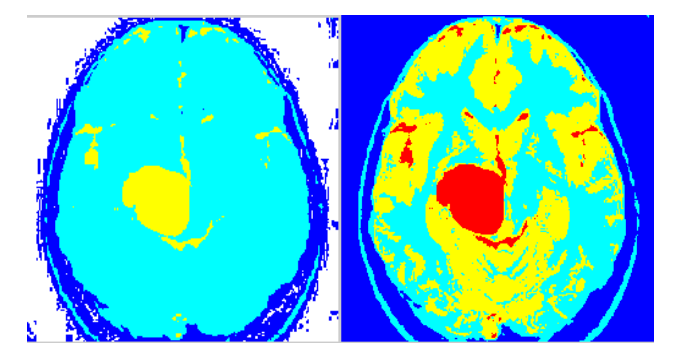

(b)

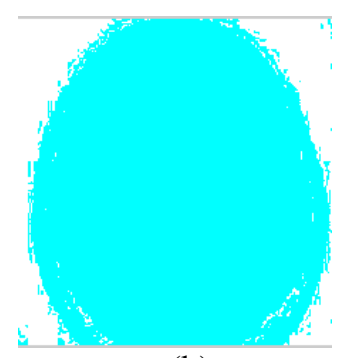

(b)
Fig. 4: A input image $(256 \times 256)$ b.Gray matter segmented at level $1(2 \times 2) \mathrm{c}$. GM,WM with tumor cell segmented at level $2(4 \times 4)$ d. WM, $\mathrm{GM}$, tumor and csf segmented at level $3(6 \times 6)$

Figure 4 shows the tested segmented images with various levels of abstractions of the MRI brain image. The original image $(256 \times 256)$ is shown in Fig. 4a-d shows the segmented image generated by neuro fuzzy technique with the level of abstraction. Here, we have achieved the white matter segmentation at level 1 of abstraction. Various tissues like white matter, gray matter and some small portions of tumor cells are segmented at level 2. The level of abstraction increases, the visibility of the different tissues are also increases.
In our implementation the segmentation of white matter, gray matter, tumor and csf at level 3 .

\section{CONCLUSION}

A neuro fuzzy based segmentation process to detect brain tumor was implemented. We studied the performance of the MRI image in terms of weight vector, execution time and tumor pixels detected and compared the results with the existing ones. A layer by layer abstraction level with fuzzy clustering technique was implemented to detect various tissues like white matter, gray matter, csf and tmor. We have achieved a higher value of detected tumor pixels than any other segmentation techniques. We have also achieved the weight vector value for the neuro fuzzy is $(6 \times 6)$ with the additional input features. The weight vector value, the number of tumor cells and the execution time will also be studied with different distance classifier technique. The change of growth rate of the tumor of the same patient analyze may also be undertaken.

\section{REFERENCES}

1. Bhandarkar, S.M. and P. Nammalwar, 2001. Segmentation of Multispectral MR images Using a Hierarchical Self-Organizing Map ComputerBased medical system CBMS 2001, Proceedings, $14^{\text {th }}$ IEEE Symposium on 26(27): 294-299.

2. Bhandarkar, S.M., J. Koh and M. Suk, 1997. Multiscale image segmentation using A Hierarchical self organizing map, Neurocomputing, 14: 241-272.

3. Parra, C.A., K. Iftekharuddin and R. Kozma, 2003. Automated Brain Tumor segmentation and pattern recognition using ANN, Computational Intelligence Robotics and Autonomous Systems.

4. Alirezaie, J., M.E. Jernigan and C. Nahmias, 1997. Neural Network based segmentation of Magnetic Resonance Images of the Brain, IEEE Trans. Nuclear Science, 44 (2): 194-198.

5. Pal, N.R. and S.K. Pal, 1993. A review on image segmentation techniques, Pattern Recognition 26(9): 1277-1294.

6. Haralick, R.M. and L.G. Shapiro, 1985. Survey ,image segmentation techniques, Computer Vision, Graphics Image Process, 29: 100-132.

7. Fu, K.S. and J.K. Mui, 1981. A survey on image segmentation, Pattern Recognition, 13: 3-16.

8. Sahoo, P.K., S. Soltani, A.K.C. Wong and Y.C. Chen, 1988. A survey of thresholding techniques, Computer Vision, Graphics Image Process. 41: 233-260. 
9. Ahalt, S.C., A.K. Krishnamurthy, P. Chen and D.E. Melton, 1990. Competitive learning algorithms for Vector quantization, Neural Networks 3 (3): 277-290.

10. Martinelli, G., L.P. Licotti and S. Ragazzini, 1990. Nonstationary lattice quantization by a selforganizing Neural network, Neural Networks 3 (4): 385-393.

11. Kohonen, T., 1988. Self-Organization and Associative Memory, 2nd Edition (SpringerVerlag, Berlin, Germany.

12. Bilbro, G., M. White and W. Snyder, 1987. Image segmentation with neurocomputers, In: R. Eckmiller and C.van der Malsburg (eds.), Neural Computers, NATO AS1 Series, (Springer-Verlag, Berlin, Germany), 41: 7 1-79.

13. DeSieno, D., 1988. Adding a conscience to competitive learning, Proceeding of IEEE the Second International Conference on Neural networks(ICNN88) 1: 117- 124.

14. Lin, W., E. Tsao and C. Chen, 1991. Constraint satisfaction neural networks for image segmentation, In: T.Kohonen, K. Mkisara, 0. Simula and J. Kangas (eds.), Artificial Neural Networks (Elsevier Science Publishers), pp: 1087-1090.

15. Naylor, J. and K.P. Li, 1988. Analysis of a neural network algorithm for vector quantization of speech Parameters, Proceeding of the 1st Annual INNS Meeting, pp: 310-315.

16. Scherf, A. and G. Roberts, 1990. Segmentation using neural networks for automatic thresholding, in: S. Rogers (ed.), Proc. SPIE Conference on Applications of Artificial Neural Networks (Orlando, FL, 1294), pp: 118-124.
17. Samet, H., 1990. The Design and Analysis of Spatial Data Structures (Addison-Wesley Pub. Co., Reading, MA).

18. Kwok, T., R. Smith, S. Lozano and D. Taniar, 2002. Parallel fuzzy c-means clustering for large data sets, In Burkhard Monien and Rainer Feldmann, editors, EUROPAR02, 2400: 365-314.

19. Gonzalez, R.C. and R.E. Woods, 2002. Digital image processing, Pearson Education, 2002.

20. Mahamed, N.A., M.N. Ahmed and A. Farag, Modified fuzzy c-mean in medical image Segmentation, in Proceedings of IEEE International Conference on Acoustics, Speech, and Signal Pocessing, Piscataway, NJUSA, 6: 3429-3432.

21. Rahmi, S., M. Zargham, A. Thakre and D. Chhillar, 2004. A Parallel Fuzzy C-Mean Algorithm for Image segmentation Fuzzy information, processing NAFIPS 04, IEEE Annual meeting, 1: 234-237.

22. Hung, M.C. and D.L. yang, 2001. An Efficient Fuzzy C means Clustering Algorithm, Data mining, ICDM 2001, Proceedings IEEE International conference on pp: 225-232.

23. Schunemann, S. and B. Michaelies, 1999. A Hierarchical SOFM for analysis of not well separable Clusters of different Feature Density, ESANN'1999, Symposium on ANN, Bruges (Belgium), pp: 21-23.

24. Ruspini, E., 1970. Numerical methods for fuzzy clustering, Information Sciences, 2: 319-350.

25. Dunn, J.C., 1973. A fuzzy relative of the ISODATA process and its use in detecting compact, well Separated clusters, Journal of Cybernetics, 3: 32-51.

26. Bezdek, J.C., 1974. Cluster validity with fuzzy sets, Cybernetics, 3: 58-73. 